\title{
Innovación educativa en tiempos de Covid-19 en el proyecto Galería Central: una experiencia de creatividad docente en comunicación del arte Educational innovation in times of Covid-19 in the Central Gallery project: a creative experience in teaching practice on the communication of art
}

\author{
Natalia MELÉNDEZ. Universidad de Málaga (España).natalia@uma.es \\ Ana SEDEÑO. Universidad de Málaga (España).valdellos@uma.es
}

Resumen: Se describe la experiencia de innovación educativa de adaptación a la gestión virtual, motivada por el confinamiento debido a la COVID-19, de la puesta en marcha de una exposición en el marco del proyecto 'Galería Central' de la Universidad de Málaga. Los resultados, tomando como material principal de evaluación las valoraciones del propio alumnado implicado, evidencian una alta implicación del grupo, con una comunicación fluida a través de la herramienta de mensajería instantánea Telegram, que analizamos especialmente, así como otras vías de comunicación virtual, incluyendo el propio producto del trabajo realizado, toda vez que la muestra se desarrolló en un blog y se difundió a través de redes sociales como Instagram, Twitter o YouTube. Asimismo, a la cohesión del grupo como uno de los factores a destacar, se añaden otros aspectos que se vieron acentuados como la autonomía en el aprendizaje y resolución creativa de problemas, la seguridad para tomar la iniciativa y hacer propuestas o debatirlas, y la colaboración tanto inter-pares como con el profesorado. De esta forma, se han superado las limitaciones de la interacción individual, en un entorno abierto, transparente y colectivo, favorecido por los instrumentos de comunicación elegidos, y por el objetivo de culminar un proyecto común a pesar de las dificultades.

Palabras clave: innovación educativa, docencia virtual, creatividad, COVID-19, Telegram.

Abstract: We describe the educational innovation experience of adaptation to virtual management, motivated by the confinement due to COVID-19, of the launching of an exhibition within the framework of the 'Central Gallery' project of the University of Malaga is described. The results, taking as the main evaluation material the assessments from the involved students, show a high involvement of the group, with fluent communication through the instant messaging tool Telegram. We analyze it especially, as well as other virtual communication channels, including the very product of the work carried out, since the sample was developed on a blog and was disseminated through social networks 
such as Instagram, Twitter or YouTube. Likewise, to the cohesion of the group as one of the factors to be highlighted, other aspects are added, such as autonomy in learning and creative problem solving, the security to take the initiative and make proposals or debate them, and collaboration both with peers and with the teachers. In this way, the limitations of individual interaction have been overcome, in an open, transparent and collective environment, favored by the chosen communication instruments, and by the objective of completing a common project despite the difficulties.

Keywords: educational innovation, virtual teaching, creativity, COVID-19, Telegram

\section{Introducción}

\section{Marco teórico: interdisciplinariedad en ciencias sociales y humanas}

Las instituciones superiores de enseñanza se han enfrentado a una importante labor de cambio de la mayoría de sus procedimientos metodológicos de la práctica y de la evaluación en las últimas décadas. De manera paralela, a la transformación cultural y logística de otros muchos procesos colectivos y públicos, se ha dejado atravesar por requerimientos nuevos procedentes de necesidades y oportunidades diferentes de los actores sociales implicados.

El conocimiento derivado de la investigación y su transmisión en los procesos docentes se ha modificado hacia una mayor complejidad, cuando no es por el tratamiento de grandes cantidades de datos, es por la hibridación de materias y disciplinas de procedencia de profesores, investigadores, estudiantes y otros. Tanto el arte y los procesos creativos como la ciencia aparecen muy alejados de la sociedad a priori. Desde la teoría crítica se ha venido denunciando cómo la diferenciación entre arte y ciencia generaba en el conocimiento un pensamiento segmentado y no plural que alejaba de la complejidad de la realidad, a cuya consciencia todo artista y científico debía aspirar.

Sin embargo, la creciente especialización en las tareas obliga a trabajos y proyectos concebidos y gestionados en equipo, normalmente transdisciplinares o multidisciplinares. Esta interdisciplinariedad entre ciencias y artes supone un desafío creciente. La universidad, la academia y todas las instituciones educativas tienen el deber de formar a sus estudiantes para aprender a pensar y adaptarse a un entorno intrínsecamente cambiante: "la universidad por venir deberá ser una institución formativa e innovadora cuyos proyectos curriculares y extracurriculares, sustentados en las perspectivas inter y transdisciplinaria, estén vinculados con el modelo productivo de la región en las diferentes áreas del conocimiento y de los saberes" (Acosta, 2016, p. 155).

No es un camino insólito, sino que ha comenzado y que triunfa en los nuevos paradigmas como el de las humanidades digitales, conjunto de técnicas y metodologías para la investigación en humanidades, en interacción con las posibilidades de herramientas informáticas. Las STEM o CTIM (Ciencias, Tecnología, Ingeniería y Matemáticas) 
suponen un enfoque que propone proyectos interdisciplinares aprovechando los elementos comunes entre las asignaturas, sus contextos y situaciones, para generar experiencias de aprendizaje e investigación significativos. Se trata de combinar el cruce de objetos y procedimientos de aprendizaje en busca de una enseñanza integradora. De hecho, en el ámbito de la comunicación ya se ha denunciado como la hiperespecialización temática está abocando a cierto tribalismo científico (Martínez-Nicolás, Saperas y Humanes, 2019, p. 29). La creación artística es una metacompetencia genérica no reconocida en este contexto. Como dice Bethania Barbosa de Souza en su artículo titulado Hibridación transdiciplinaria en las artes plásticas, la creación artística supone "los modos de expresar, de interpretar y entender el proceso creativo ya no se encuentran arraigados a una disciplina o técnica específica, aislada de las demás disciplinas como solía ocurrir anteriormente" (Barbosa de Souza, 2009).

A pesar de esta situación en educación e investigación de creciente transdisciplinariedad, en comunicación se viene detectando un descuido teórico y práctico de ciertas subáreas como las de guión, realización audiovisual o creación intermedia y transmedia en la vertiente de producción es uno de los conflictos de la formación en comunicación, especialmente en la relación con el alumnado. En este sentido e intentando paliar este contexto, el proyecto Galería Central trata de compilar en una misma actividad elementos de variadas disciplinas, poniendo al servicio de la creación artística las capacidades comunicativas, tanto desde el ámbito de la publicidad, como de la gestión y atracción de públicos a través de las redes sociales, como desde la logística y la organización curatorial.

Además, los ámbitos del arte y de la comunicación comparten ciertas lógicas de producción de textos o productos de creación/artísticos y audiovisuales. La obra (tanto artística como comunicativa) es esfuerzo de acción, de reflexión, de pensamiento y tiene un carácter performativo o locucional en algunas ocasiones, otras factual y otras establece además una vertiente de exteriorización, en el caso de los productos comunicativos, de naturaleza mediática. En cualquier caso, en ambos ámbitos se trabaja con ideaciones, conceptos que se convierten en productos con diversos formatos de salida (Sedeño-Valdellós, 2019, p. 10). Y en este contexto, el alumnado de las facultades de comunicación estudia unos objetos comunicacionales con una naturaleza artística, que deben saber apreciar y transmitir, hasta tal punto que implica un tipo de periodismo especializado que necesita de fuentes y documentación (Izquierdo Expósito \& Fernández Maestre, 2019). Son agentes de mediación artística (Rodrigo-Montero, 2015) y por ello esta labor de comisariado es parte de una hibridación disciplinar creciente: "Este proceso de transgresión está generando un enriquecimiento en el proceso creativo, ampliando los horizontes creativos no sólo de los artistas, sino también del alumnado en todas las etapas de su educación" (Barbosa de Souza, 2009, p. 219).

Otro cambio de paradigma supone el giro artístico (Buck-Morss, 2004; O'Neill \& Wilson, 2010), cuyo principal objetivo operativo explícito ha convertido a la educación artística en un propósito educativo global, casi en una competencia general en todos los niveles educativos. La Unesco, en respuesta a su promoción de la educación artística y la creatividad, ha organizado conferencias mundiales como la de Lisboa en 2006, "Hoja de 
ruta para la Educación Artística" o la de Seúl en 2010, "Agenda de Seúl: Objetivos para el desarrollo de la Educación Artística" (UNESCO, 2006).

En el trabajo pretendemos presentar una experiencia de mediación artística y comisariado durante la pandemia por COVID-19, a través del trabajo colaborativo con una herramienta como Telegram para la organización y gestión del espacio expositivo Galería Central, en la Facultad de Ciencias de la Comunicación de la Universidad de Málaga. El proyecto era un ejemplo vivo de la necesidad de saltar las fronteras entre las disciplinas, y de acercar a colectivos universitarios, docente y estudiantes el mundo elitista del arte. $\mathrm{Su}$ necesidad de seguir formando en mediación artística y comisariado desde la experiencia a través de proyectos de exposición condujo a la búsqueda de soluciones de virtualización en un contexto de incertidumbre e improvisación y adaptación como fue el derivado del confinamiento decretado tras el estado de alarma en marzo de 2020. Es decir, el trabajo presenta los resultados de una doble innovación, por una parte la que ya de por sí suponía esta experiencia, sumada a la derivada de las circunstancias sobrevenidas por la crisis sanitaria.

\section{Tecnología y adaptaciones de la enseñanza y la práctica de la mediación artística después del Covid-19}

La tecnología tiene muchos aspectos problemáticos que se manifiestan en el territorio educativo, campo de batalla de todo tipo de retos culturales y sociales: el ciberacoso, los virus, la desinformación y los bulos, el sexting y otros aspectos éticos de su uso relacionados con la intimidad, la privacidad... están presentes y si hablamos de todo tipo de vulnerabilidades que multiplican su efecto (Prendes y González, 2020), el riesgo es mayor. Pero este discurso se ha visto modificado por muchas razones, como sería muy extenso describir aquí.

El 13 de marzo de 2020 comenzaba la situación de estado de alarma por Covid-19 en España. No sólo había que adaptar la enseñanza sino también todas las prácticas de investigación, gestión y transferencia de la universidad y demás instituciones universitarias. Y ello no sólo desde el punto de vista del profesorado sino del alumnado y de todas las relaciones de estos con los estamentos y agentes implicados. En términos de docencia, se ha hablado de docencia virtual, remota, bimodal, no presencial y otros muchos términos. Hodges, Moore, Lockee, Trust \& Bond (2020) lo han denominado docencia remota de emergencia (DRE) (en inglés emergency remote teaching), y lo han definido como:

un cambio temporal de provisión de la enseñanza a uno alternativo debido a circunstancias de crisis. Implica el uso de soluciones totalmente remotas para la instrucción o la educación, que de otro modo se ofrecerían de modo presencial o como cursos mixtos o híbridos y que volverán a ese formato una vez la crisis o emergencia haya remitido (Hodges, Moore, Lockee, Trust \& Bond, 2020, p. 6).

También Jiménez \& Ruiz (2021) han reflexionado acerca de los desafíos específicos sobre la educación superior en términos de limitaciones en el espacio y el tiempo en que deben 
producirse las adaptaciones de todos los agentes y procesos educativos durante la pandemia. Gasca Herrera (2020) concluye que los profesores tienen suficiente infraestructura tecnológica para impartir clases en línea aunque la incertidumbre sobre los modelos de enseñanza a largo plazo arriesgan la planeación académica.

En esta situación de pandemia mundial, hay que asumir que formar desde el hecho de que el alumnado sea dueño de su formación implica que la creatividad y la imaginación en la resolución de problemas es educable y puede suponer la supervivencia de proyectos educativos pequeños (Lin \& Reigeluth, 2021). La tecnología nos permite gestionar lo no presencial, permite la virtualidad para aplicarla a todas las fases de la creación de la mediación artística que suponen las exposiciones de arte. Ser creativos en este contexto supone la resolución de problemas y situaciones no experimentadas anteriormente y tomar decisiones apropiadas en situaciones de adversidad (Cortes \& Márquez, 2017).

\section{Galería Central: breve presentación}

Galería Central es el espacio expositivo de la Facultad de Ciencias de la Comunicación de la Universidad de Málaga (UMA), en el edificio que comparte con la Facultad de Turismo. El día 20 de noviembre de 2008 se inauguró oficialmente esta galería universitaria. Hablamos, pues, de un proyecto consolidado, que cuenta con 13 años de andadura pero que por primera vez se enfrentó a la necesidad de virtualizar todo el conjunto de tareas que lo hacen posible.

Desde sus inicios, los objetivos que se han querido conseguir con el proyecto en los ámbitos de la enseñanza-aprendizaje y de la organización docente son los siguientes (Lumbreras, Garnelo \& Gabilondo, 2011):

- Consolidar la Facultad de Ciencias de la Comunicación dentro de la ciudad de Málaga, acercando esta institución educativa a los malagueños.

- Constituir un foro permanente de debate sobre el trabajo creativo en Comunicación.

- Hacer más visible y accesible la producción artística e innovadora de la comunidad universitaria, abriendo nuevas vías de diálogo con la realidad cultural malagueña.

- Crear una red de universidades españolas que facilite la gestión de proyectos conjuntos y el intercambio de experiencias singulares.

- Promover el contacto con los profesionales del sector, trabajando con estos colectivos en la consecución de una mayor creatividad en la comunicación.

Como vemos, uno de los principales objetivos de Galería Central consiste en "constituir un foro permanente de debate sobre el trabajo creativo en Comunicación" y que esto se convierta en un principal eje para la enseñanza transdisciplinar ${ }^{1}$ en ciencias sociales. Así pues, desde 2013, esta iniciativa se desarrolla dentro de un Proyecto de Innovación Educativa (PIE) en

1 Con este término entendemos un paradigma educativo que aúna lo inter-, multi- y transdisciplinar, concebidos como integración completa de disciplinas del conocimiento, que en este caso también debe ser comunicado a través de una experiencia educativa. 
gestión y comunicación de proyectos culturales, gracias al Vicerrectorado de Innovación Educativa de la UMA, con apertura y colaboración a otros centros de la Universidad de Málaga, como Arquitectura, Bellas Artes y Turismo, que contienen estudios de ámbito multidisciplinar, en torno a los distintos aspectos de lo artístico y cultural. Se emplea este contexto con objetivos educativos a modo de creación e investigación desde la práctica, con la meta de la producción de obras y proyectos, generando todo lo necesario para su visibilización, exhibición y recepción en un espacio público. Ese espacio público es educativo porque se instaura en una institución universitaria, pero conforma un contexto de trabajo que se expande especialmente durante las al menos dos semanas previas a cada inauguración.

Galería Central se centra en proyectos educativos bajo metodología de PBL (Aprendizaje por proyectos), de manera congruente con el espacio Europeo de Educación Superior, un proceso de trabajo en equipo, que prima la autonomía dentro de unas responsabilidades específicas. El PBL tiene tres dimensiones temporales que se concitan plenamente en Galería Central:

Facilita la motivación al presentar situaciones problematizadas que el estudiante explora a partir del conflicto cognitivo; activa el aprendizaje desde un entorno en el que las interacciones tienen lugar entre los miembros del propio grupo de estudiantes y entre éstos y el equipo docente; y finalmente, se retroalimenta constantemente al estudiante por parte del docente, tanto en las sesiones de evaluación como en el trato personal, a través de las tutorías. En cualquier caso, la propuesta de formación basada en problemas pretende afrontar aspectos como los siguientes: Otorgar relevancia (social, profesional, académica) a las situaciones de aprendizaje; Implicar y fortalecer el punto de vista del estudiante en el aprendizaje; incrementar el sentido de autonomía en el aprendizaje; generar situaciones en las que se requiere un enfoque socioconstructivista para desarrollarlo; desarrollar competencias para el aprendizaje y recursos para el mismo. (Rué, Font \& Cebrián, 2011, p. 27)

En este contexto descrito desde hace ya casi dos décadas, se presupone que el alumnado es el autor de su aprendizaje y que en la enseñanza superior tiene que culminar su formación en competencias para un aprendizaje a lo largo de la vida. El profesorado como facilitador debe generar experiencias y prácticas significativas para materializar contextos donde esto se haga posible.

\section{Material y métodos}

En el presente trabajo pretendemos exponer cómo se ha desarrollado la puesta en marcha de la exposición virtual 'Diamantes en bruto' (ediciones X y XI, correspondientes a materiales de los cursos 18/19 y 19/20), inaugurada el 25 de septiembre de 2020 y cómo se adaptó completamente el sistema de trabajo de Galería Central a la circunstancia de no poder mantener reuniones presenciales ni utilizar el espacio físico habitual del centro para poder desarrollarla. Con ellos pretendemos demostrar cómo la creatividad se puso al servicio de una situación sobrevenida de docencia remota de emergencia y cómo nuestro estudiantado fue capaz de resolverla mediante la virtualización de las 
operaciones necesarias para completar con buen fin la tarea a acometer. Así pues, más que de materiales y métodos, hablaríamos sobre todo de descripción de la experiencia y contexto de desarrollo, planteando que nos encontramos ante una metodología cualitativa en la que se ha utilizado como fuente para la investigación un cuestionario a las alumnas responsables de comisariar la exposición, completado con las valoraciones de las acciones emprendidas expresadas de manera informal por todos los implicados en conversaciones de Telegram -más abajo abundamos en su uso docente- mantenidas por el grupo de trabajo. Asimismo, estos frutos de la evaluación formales como el cuestionario e informales como las conversaciones de Telegram a modo de diario de campo, se ven completados con el resultado final de la puesta en marcha de la exposición, con los datos de difusión y seguimiento de la misma. Todo ello nos habla, como veremos, de un caso de éxito que juzgamos podría tener la utilidad de servir como guía en experiencias similares.

Llegados a este punto, expondremos la sucesión de las diferentes fases experimentadas en este proyecto. En el momento en el que se declara el estado de alarma acababa de ser seleccionado el grupo de estudiantes que formarían parte del equipo para el periodo de dos años de duración del PIE concedido para seguir realizando la labor de Galería Central. Los docentes coordinadores seleccionaron a estudiantes de las titulaciones de Periodismo, Comunicación Audiovisual y Publicidad y Relaciones Públicas, procurando el equilibrio en habilidades y conocimientos previos para poder llevar a cabo las tareas propias de una labor de comisariado, montaje y difusión de una galería artística. Sin embargo, la primera reunión presencial para asignar los diferentes cometidos tuvo que anularse debido al confinamiento.

Después de un periodo de incertidumbre y adaptación a la nueva situación desde los distintos órganos implicados, se decidió retomar la actividad, en este caso transformada en comunicación estrictamente virtual, como así lo sería finalmente el producto final de los preparativos para la exposición 'Diamantes en Bruto'. Dicha exposición supone un encuentro esperado de Galería Central ya que consiste en la muestra anual de trabajos procedentes de prácticas de clase del propio alumnado de diversas asignaturas. Se trata, pues, de una exposición colectiva que cumplía en 2020 su décima edición.

De este modo, se contactó con el alumnado por correo electrónico para indicarles que se mantendría una primera reunión virtual a través de Google Meet en la que se explicaron los objetivos inmediatos, se asignaron tareas (redacción, diseño, mantenimiento de redes, manejo de equipamiento audiovisual...) y se decidió apostar por una nueva modalidad de exposición, en esta ocasión lejos de las paredes del centro como hasta entonces, para centrarnos en el proyecto de una muestra completamente online. Con posterioridad se efectuaron varias reuniones virtuales más, pero lo que agilizó enormemente el trabajo fue decidir prescindir del correo electrónico y usar una comunicación mucho más inmediata a través de la mensajería instantánea en el móvil vía Telegram, creando un grupo de 14 personas, que permitía realizar búsquedas por tipo de contenido, así como realizar encuestas o enviar archivos de hasta 1,5Gb (Figura 1). 


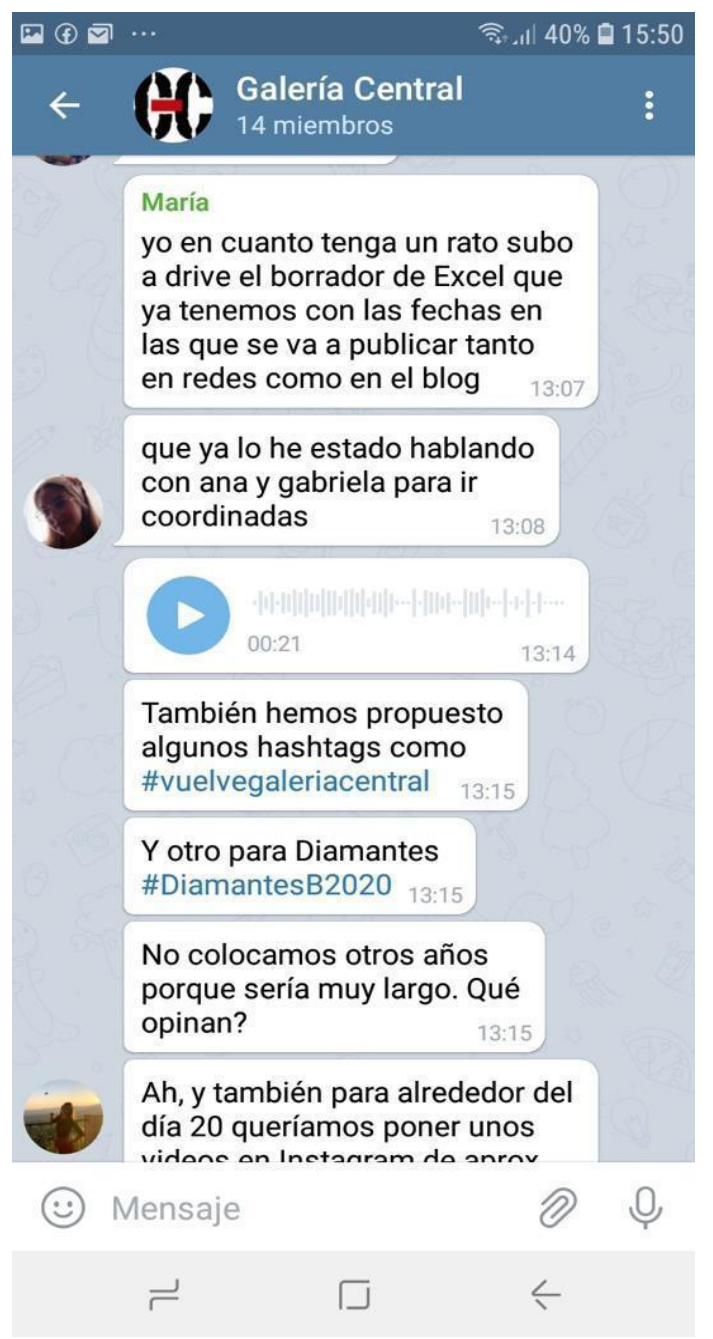

Figura. 1 Captura de pantalla del grupo de Telegram usado como método de comunicación habitual.

Varios han sido los trabajos académicos que profundizan en las utilidades de la aplicación como forma de lo que se ha denominado Mobile Learning a través de Telegram. Faramarzi, Heidari \& Chalak (2019) describen las funciones y ventajas como la red perfecta de acceso a canales y grupos y de intercambio y lectura de todo tipo de archivos y ponen el foco en sus características y su adaptabilidad. Salas y Salas (2018) destacan las propiedades de socialización ligadas al aprendizaje colaborativo:

el uso de la herramienta Telegram incide en la participación y la socialización, promoviendo el aprendizaje colaborativo e incidiendo en el comportamiento, permite reconocer a las diferencias individuales, aumenta el desarrollo interpersonal, se logra que el estudiante interiorice en su aprendizaje y apoye al grupo lo que le da sentido de logro y pertenencia e incremento de la autoestima. (Salas y Salas, 2018, p. 90)

Frente a Whatsapp, Telegram es fácil de manejar y todos los miembros del grupo actúan como administradores, lo que redunda en una colaboración más horizontal y participativa: 
el aprendizaje móvil de Telegram es flexible para profesores y estudiantes en función de los comentarios recopilados de los participantes. Los comentarios de los participantes también muestran que el uso de Telegram de aprendizaje móvil brinda una nueva experiencia, ya que es creativo, interesante y espontáneo. Los participantes también declararon que disfrutaron aprender a través de Telegram porque se sentían más relajados y se sentían cómodos de participar activamente en compartir su punto de vista honesto. (Iksan \& Mohd, 2017, p. 22).

También Franco Campoverde y Malagó Rosado (2021), Kasa y Yusuf (2020) o Estrada Molina, Fuentes Cancel y García Hernández (2021) inciden en experiencias específicas gracias a las posibilidades para la creación de grupos y canales, envío de actividades interactivas a estudiantes como ventajas importantes, unidas al menor peso de la aplicación y la seguridad como elementos diferenciadores que empujan su popularidad entre estudiantado y profesorado. De hecho, todas estas ventajas son las que se han ido descubriendo durante la gestión del comisariado virtual, como expondremos en el apartado de Resultados con citas paradigmáticas.

De este modo, Galería Central trató de adaptarse como espacio universitario al concepto de "presencialidad adaptada" del que hablan la mayoría de documentos de instituciones educativas españolas durante la situación de estado de emergencia y el confinamiento asociado (Ministerio de Universidades, 2020). Aurea-Moreira, Bethencourt-Aguilar, Martín-Gómez, \& San Nicolás-Santos (2020) explican que este concepto es "una distorsión de las características y principios pedagógico-organizativos del blended learning o enseñanza semipresencial" (p. 15) y que estos no se han tenido en cuenta durante estos meses en la enseñanza universitaria en España.

Por el contrario, quizás proyectos de innovación como el de Galería Central, por sus labores de comisariado, curaduría o mediación artística pueden ser gestionadas desde lo virtual, con la condición de que se produzcan con un soporte mediado, ya sea el espacio expositivo físico o algún medio de difusión/exposición con el que llegar al espectador.

La labor del comisariado o curaduría supone tareas que están imbricadas en la concepción actual del arte contemporáneo, en su paradigma de posproducción (Bourriaud, 2004), donde las muestras y las exposiciones son discursos sobre obras creativas, que están en permanente circulación y que con las posibilidades tecnológicas incrementan su tiempo de exposición al público durante meses y años, cuando no son permanentes en blogs y páginas webs. Además, la mayor parte de la discusión que ha generado desde perfiles académicos y artísticos coinciden en que tiene funciones de mediación artística, metaautoría (Feldman, 2012; Koldobsky, 2009), de transferencia del conocimiento y funciones creativas que actúan con una visión legitimadora de artistas y campos artísticos, de ahí su influencia y relevancia. Las competencias asociadas a la curaduría incluyen, además, las de organización y, como resulta fácil de razonar, se aprenden "en el campo", sobre el terreno de la relación con los artistas, el espacio, los materiales y todos los procesos para organizar una exposición:

El comisariado de exposiciones de arte realiza un intenso trabajo de investigación, documentación y análisis crítico de las obras, los/as artistas y sus contextos históricos y actuales. (...) Respecto de una transferencia del conocimiento artístico en la exposición de arte, se entiende que las formas de exponer el arte son deudoras de los modos de pensarlo. (Tirado, 2019, p. 169) 
Las labores de mediación artística contienen, por tanto, una doble naturaleza pues requieren organización, reuniones y toma de decisiones para realizar labores de selección de obras y artistas, así como de reparto del espacio y la experiencia visual/vital del visitante de la exposición. De igual modo, se suma que las obras son de distinta naturaleza y fisicidad (formato, dimensiones, duración...). Como cuenta Sanz Daza en Manual del Comisario del Catálogo de Galería Central 2012-2014:

Ser comisario de una exposición consiste en ser el máximo responsable de la misma. Esto quiere decir que el resultado final depende directamente de él. Debes tener cuidado, sin embargo, de no confundir esto con echarse todo el trabajo encima. El comisario es el director de la exposición, el que se encarga de que todo tenga armonía y cohesión. Como director, su labor es mandar al resto del equipo que haga diversas tareas. Por supuesto, el propio comisario puede realizar las tareas él mismo, pero no es aconsejable que sea esta figura la que cargue con todo el grueso del trabajo; debemos recordar que somos un equipo, y que por tanto, todo el mundo debe participar e implicarse. Aprender estas destrezas y habilidades de gestión, planificación y de presentación de una propuesta de muestra artística son ya complejas en un contexto presencial: todo ello debía adaptarse para que los alumnos siguiesen su formación y la posibilidad de que sus compañeros y toda la comunidad universitaria conociese su trabajo. (Sanz-Daza Pérez, 2018, p. 14)

Estas funciones complejas y tan abiertas de organización, gestión, contacto, promoción, etc. debían ser adaptadas, así como la muestra física específica en sí. En este sentido, la tarea fundamental fue la de recopilación del material y las decisiones en torno al diseño y la presentación de las obras. Así, se realizaron, especialmente, tareas de rediseño del blog en Wordpress del proyecto, soporte que albergaría la exposición virtual. Dos alumnas fueron las encargadas de comisariar la exposición. Su labor duró varias semanas, en las que hubieron de contactar con el profesorado de las asignaturas tradicionalmente vinculadas a la exposición 'Diamantes en bruto', que, por su carácter más práctico, generan gran cantidad de obras susceptibles de ser expuestas. Más tarde, hubieron de contactar con las y los estudiantes autores de cada obra para solicitar su permiso y obtener el material en la mejor calidad posible. De este modo, recabaron información sobre las obras, sobre cómo preferían que se presentaran en el blog y redactaron textos generales para su difusión (nota de prensa, entrada en el blog...) y textos explicativos a modo de cartela ampliada. De su difusión se encargó otro equipo que gestionaba las redes, por un lado, y YouTube, por otro. De esta forma, una vez asignadas responsabilidades de acuerdo con el perfil del alumnado, se pudieron reactivar las redes sociales de Galería Central (Instagram, Twitter, canal de YouTube), fundamentales en la virtualización del proyecto.

En el caso de redes, Instagram y Twitter, se generó material para anunciar la exposición y su inauguración, incluyendo, a iniciativa del propio alumnado, stories y reels de Instagram con los propios artistas invitando a asistir y a ver la muestra.

En el caso de YouTube, su papel fue clave para poder realizar a través de un directo la inauguración (un acto de carácter institucional en toda regla en el que se contó con la presencia de las alumnas comisarias, la decana del centro, la vicerrectora de Cultura y las docentes coordinadoras de Galería Central), se diseñaron los rótulos de las personas intervinientes, así como fondos para la cuenta atrás antes del inicio del directo y un vídeo- 
resumen de las obras. Este trabajo realizado totalmente en equipo, pretendió desde el principio presentar un acabado plenamente profesional y la retransmisión en streaming alcanzó un gran dinamismo toda vez que se preparó meticulosamente con antelación, y hubo comunicación continua a través de Telegram mientras se desarrollaba. Su resultado final puede verse en el canal de YouTube de la Facultad de Ciencias de la Comunicación de la Universidad de Málaga (https://www.youtube.com/watch?v=cqqTLUfeKVs).

\section{Resultados y discusión}

En Galería Central, superado el estado de confinamiento por la COVID19 durante el curso 19/20 que afectó a la planificación temporal del mismo, se pudo reanudar la actividad con el comienzo del nuevo curso 20/21. Una vez retomado el ritmo de actuaciones, las rutinas de trabajo están fijadas y la experiencia del alumnado se deja ver en la soltura con la que ya se realizan muchas tareas, ya sí planeadas, para las exposiciones que han seguido a esta primera experiencia piloto no planificada. Exponemos aquí algunos de los resultados.

Montaje completo de la exposición virtual 'Diamantes en bruto' en septiembre de 2020 (a la que han seguido dos presenciales en octubre y noviembre de 2020). Diamantes en bruto $X$ (2018-2019) contó con un total de 67 obras expuestas, de las cuales 56 pertenecen a la titulación de Comunicación Audiovisual, 9 a Publicidad y RRPP y 8 a Arquitectura. Y en cuanto a los formatos, 5 fueron en vídeo y 62 consistieron en fotografias. Diamantes en bruto XI (2019-2020) contó con un total de 131 obras expuestas, de las cuales 76 pertenecen a la titulación de Comunicación Audiovisual, 3 a Publicidad y RRPP y 52 a Arquitectura. Y en cuanto a los formatos, 12 fueron en vídeo y 119 consistieron en fotografías.

Se ha trabajado con normalidad en este comisariado virtual tras la adaptación que se ha comentado:

El comisariado virtual requirió muchas horas delante del ordenador para coordinar la selección de obras y su disposición en el blog (que cumplió en esta exposición la labor del espacio virtual en el que se pueden ver las obras de manera atemporal). Estas dos tareas fueron para mí las actividades más complicadas de hacer, que si no hubiera habido covid se habrían traducido en horas de cafés y "debate" sobre las obras en la facultad. Se solucionaron con mucha comunicación y siendo tanto Gabriela como yo, flexibles en la toma de decisiones y de observaciones por parte de otros miembros de Galería. (Ana, alumna comisaria de Diamantes en Bruto X y XI).

Estas palabras parecen evidenciar un cierto desplazamiento en esta generación sobre la idea de que pese a la imposición de las tecnologías digitales como forma de comunicación, en según qué ocasiones las personas siguen prefiriendo el «cara a cara» (Mansell, 2003). La presencia física aquí sin embargo no ha sido reclamada como una carencia en esta experiencia.

El blog de Galería Central no solo recoge la exposición virtual 'Diamantes en bruto' sino que se actualiza con regularidad y contiene además un apartado en el que se listan todas las exposiciones realizadas históricamente, también incluye la repercusión en medios, 
que consideramos un resultado importante de este trabajo. Puede consultarse en https:// galcentral.wordpress.com/gabinete/ y podemos destacar que gracias a la redacción y envío de notas de prensa, las inauguraciones de exposiciones han aparecido en la agenda cultural de Hoy por hoy Cadena Ser Málaga, la portada de la web de la UMA o la revista El Giraldillo, que tiene 50.000 visitas diarias.

Otro resultado relacionado con la difusión es la actividad en medios sociales: las cuentas en redes han alcanzado los 2.154 seguidores en Twitter y 483 en Instagram. El vídeo en YouTube con la inauguración virtual ha obtenido, de momento, 120 visualizaciones.

Considerando los retos que ha habido que afrontar, juzgamos que el proceso recorrido hasta ahora es satisfactorio y ha resultado en un aprendizaje añadido para el estudiantado, al desarrollar estrategias creativas para superar los imprevistos de una situación sobrevenida haciendo uso de las nuevas tecnologías y nuevos procesos (comunicación interna a través de un grupo de Telegram y videollamadas, retransmisiones en streaming, comunicación en redes sociales, creación y explotación de un blog en modo "sala de exposiciones" (https://galcentral.wordpress.com/), así como la gestión de relaciones con los medios especializados). Igualmente, se ha llevado a cabo un proceso de aprendizaje inter-pares, donde estudiantes de diferentes cursos y grados han trabajado conjuntamente para sacar adelante sus tareas individuales para llegar al objetivo conjunto final de la exposición, donde han asumido un rol protagonista como comisariado de la muestra con el apoyo de los docentes que han asumido un papel de promotores de este aprendizaje:

Pues creo que aprendí bastante de Gabriela, en cuanto a su percepción de las obras y de cómo ella consideraba que el público las percibiría. Ella es más de imágenes y yo más de palabras, por lo que creo que hicimos muy buen equipo. También como he mencionado antes, el tema de "ser flexibles" y no querer imponer tus ideas porque sí, sino que todo tuviera una lógica. Además, aprendí el hecho de pensar en un objetivo que no sea "sacar buena nota en una asignatura", pues en este caso la idea era sacar adelante un proyecto que implica a muchas otras personas y a la propia facultad a la que pertenezco. (Ana, alumna comisaria de Diamantes en bruto X y XI).

La satisfacción del trabajo finalizado se hace patente en las propias reacciones de los integrantes del grupo, como esta que utilizamos a modo de ejemplo. "Muchas gracias a todo el equipo. La verdad es que ha sido genial poder exponer otra vez en Galería” (Rocío, estudiante de Máster).

La colaboración y buena disposición de las y los estudiantes ha sido fundamental para el buen funcionamiento de Galería Central en un periodo marcado por la incertidumbre generada por la COVID19, que ha supuesto modificar las pautas establecidas y la hoja de ruta inicial. Apreciamos, pues, que su flexibilidad y adaptabilidad a las circunstancias han sido también un ejemplo de creatividad aplicada en este caso a la solución de problemas. Saber a veces improvisar ante la adversidad, tomar decisiones en poco tiempo, todo ello ha formado parte de la experiencia:

Este año hubo muchas peculiaridades, la más importante es que fue la primera exposición únicamente virtual de Galería Central, entonces hubo varios retos para adaptar el número 
de obras, las asignaturas y las titulaciones. De todas formas, Ana y yo pudimos encontrar la forma de llegar a un acuerdo con el equipo, los artistas y con Wordpress. Aprendimos mucho de gestión, y lo más importante, le dimos el protagonismo que merecían a los alumnos. (Gabriela, alumna comisaria de Diamantes en Bruto X y XI).

Encontramos aquí correspondencia con lo sugerido por Franco Campoverde y Malagó Rosado (2021) acerca de que los alumnos tienen que aprender de manera colaborativa, enfrentándose a problemas de logística y organización reales y con tiempos en contexto según las tareas a realizar. En una situación como la del confinamiento por Covid-19, el aprendizaje que se debe buscar es el de un "modelo educativo a distancia, aquel que sea autónomo y autorregulado, para favorecer el logro de aprendizajes significativos".

Pero no solo para el alumnado, sino también para el profesorado, el proceso ha sido enriquecedor, al provenir de diferentes especialidades los coordinadores han aprendido también del área de sus colegas y del propio alumnado, de quienes recogían propuestas con naturalidad, en ese foro común que se establece a través de la comunicación colectiva en este caso vía Telegram (figura 2). Así pues, no es un estudiante en concreto el que presenta una duda o escollo y se le ayuda a superarla con sugerencias desde una interacción individual, como ocurriría mediante el correo electrónico o en tutoría presencial, sino que todo el grupo asiste a cómo se resuelve ese problema y aprende aunque no sea el generador de dicha consulta, e incluso a veces se decide de manera asamblearia la solución. El alumnado destaca esta relación inmediata y este aprendizaje colectivo y paralelo al profesorado:

El asesoramiento de los profesores fue clave para que saliera adelante el proyecto, es como una tutoría virtual con mejor interacción. Como tienen mejor conocimiento de la Facultad en términos generales nos decían qué podía hacerse, qué no, etc. Siempre contestaban y eso nos ayudó a verificar si el trabajo iba bien. Se agradece mucho, esperamos que la comunicación a través de Telegram siga así. (Ana, alumna comisaria de Diamantes en Bruto X y XI).

Respecto al papel de Telegram, parece que supuso una herramienta perfecta para la organización y comunicación, siempre de manera flexible y complementaria a otros recursos:

el asesoramiento nos permitió sobre todo seleccionar entre todas las obras, porque el hecho de tener espacio ilimitado en el blog nos hizo creer que más obras aportarían más, cuando realmente podría ser algo contraproducente. Además, siempre nos ayuda que otras personas, que tienen más experiencia que nosotras, nos asesoren en el lenguaje utilizado, para que sea inclusivo, y por supuesto sencillo. Además, el hecho de hablar por Telegram nos hacía ir más al grano. (Ana, alumna comisaria de Diamantes en bruto X y XI).

Como vemos, paradójicamente, lo virtual, que a veces parece aislarnos, humaniza cuando se trabaja en la resolución de problemas, algo que fomenta el trato a veces más relajado con los códigos de las redes, como el uso de emojis, gifs o stickers, que es también, no lo olvidemos el lenguaje cotidiano de nuestro alumnado:

Telegram permitió que en cuestión de segundos enviáramos una foto o algún archivo, se revisara y si había que cambiar algo sencillamente lo decíamos y se corregía, el tiempo no se perdía. Todos podíamos participar y si no se podía inmediatamente, el mensaje o nota de 
voz quedaba allí para cuando pudiéramos revisarlo. Además, podíamos comunicarnos con stickers, ¿qué más se puede pedir? (Gabriela, alumna comisaria de Diamantes en Bruto X y XI).

Esto último va en consonancia con la propuesta de Chipia (2013), quien afirma que

La Educación del presente debe apoyarse en las Tecnologías de la Información y Comunicación y en particular de las redes sociales virtuales, para desarrollar el aprendizaje creativo, crítico y emocional de los actuales estudiantes (...). Es fundamental, en la actual sociedad, gestionar el cambio de ocio en las redes sociales virtual, en cuanto a transformar el ocio digital en ocio productivo, como una actividad motivadora, enriquecedora y llena de satisfacción personal y grupal de los participantes. (Chipia, 2013, p.138)

La autonomía en la toma de decisiones y la colaboración entre el alumnado de manera independiente es otro de los resultados que destacamos de la experiencia: “(...) ya lo he estado hablando con Ana y Gabriela para ir coordinadas "(María, estudiante de Periodismo, sobre la elección de hashtags para las redes sociales). También se hacen conscientes de en qué pueden ayudar y ser ayudados por un compañero, y de la confianza que les supone ese respaldo: "Sí, yo de directos tampoco sé mucho. Espero que contemos con ayuda de Jesús. Igual no creo que sea tan complicado" (Gabriela, comisaria de Diamantes en Bruto $\mathrm{X}$ y XX, sobre su intervención como comisaria en la inauguración virtual). La voluntad de apoyar a las y los compañeros, además de pedirles ayuda, se evidencia: "Yo misma puedo hacer la escaleta, que Gabriela estará muy saturada" (María, estudiante de Periodismo, sobre la preparación de la inauguración virtual). Todo ello conduce a la cohesión del grupo: "Mis felicitaciones a todas las implicadas (...) Jesús, no le he visto ni un fallo al directo, trabajazo" (Hugo, estudiante de Publicidad y RR. PP., tras finalizar la inauguración virtual). Se cumple aquí, pues, en nuestra valoración lo manifestado por Ovejero (1988) acerca de que en este tipo de metodologías colaborativas el alumnado permite desarrollar un mayor nivel de tolerancia, respeto y cooperación al tener que consensuar diferentes puntos de vista, creando un clima de progreso y cohesión para el desarrollo de acciones y metas comunes. Asimismo, sostenemos que estas características se fomentan especialmente en la experiencia virtual ya que la comunicación resulta más horizontal debido a unas condiciones similares de participación (básicamente fundamentada en el texto escrito) que elimina condicionantes de la interacción física (tono de voz, gestualidad, actitud corporal autoritaria...).

Galería Central, como tantos otros proyectos de innovación paralelos a la docencia y las actividades presenciales de clase en la universidad, suponen una materialización del compromiso de todos los agentes con la formación integral del alumnado. La educación artística tanto en su producción como en su gestión es una oportunidad para crear situaciones de generación de conocimiento que propicien el aprendizaje en la práctica, en un entorno lo más cercano a lo experiencial. El aprendizaje horizontal, la supervisión del profesorado, la atención a los ritmos de las y los compañeros, el descubrimiento de las posibilidades que ofrece la tecnologías y redes sociales, son los ingredientes necesarios pero deben ser gestionados a muchos niveles en diversos momentos, y deben tener como condición la actitud de continua voluntad de colaboración y servicio, especialmente en momentos de transformación profunda o de crisis, en los que nos enfrentamos a la incertidumbre. Para 


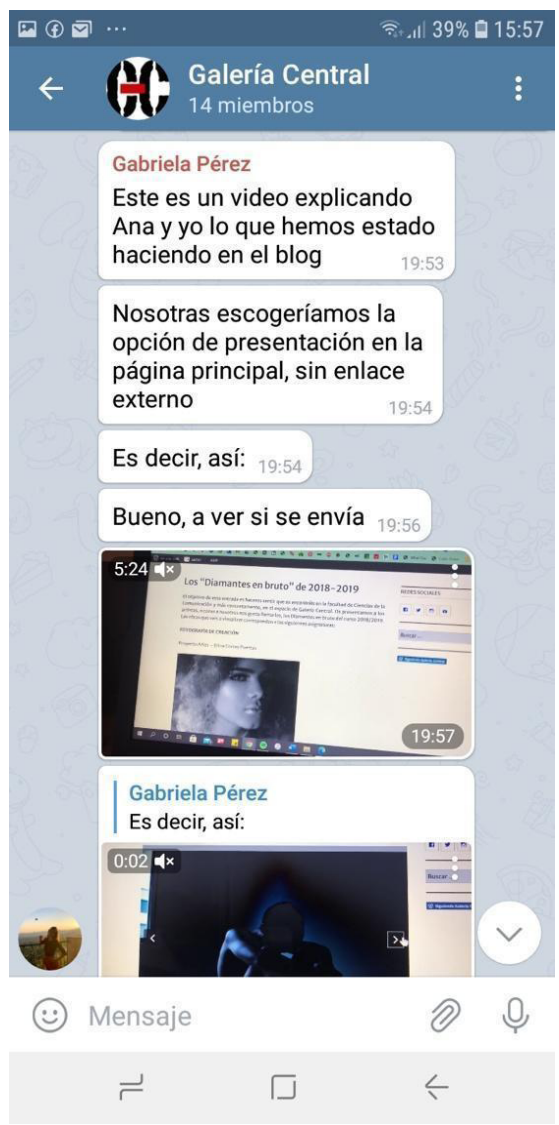

Figura 2. Captura de pantalla en la que se observan las posibilidades de mostrar el avance del trabajo y modificarlo sobre la marcha.

finalizar, las palabras de una de las alumnas involucradas, verdaderas protagonistas de la acción, resumen el resultado conjunto de la experiencia:

Es increíble que a pesar de que el 100\% de la gestión fue virtual, se llevó a cabo exitosamente. Esto quiere decir que, si hay compromiso por parte del equipo, se puede sacar un proyecto adelante. Cada uno se pudo adaptar y sacar lo mejor de sí en el área que más le llamaba la atención para que encajaran las piezas, incluidos los profesores que siempre nos estuvieron apoyando. (Gabriela, alumna comisaria de Diamantes en bruto X y XI).

\section{Referencias bibliográficas}

Acosta, J. (2016). Interdisciplinariedad y transdisciplinariedad: perspectivas para la concepción de la universidad por venir. Alteridad. Revista de Educación, 11(2), 148-156. doi: 10.17163/alt.v11n2.2016.01

Aurea-Moreira, M., Bethencourt-Aguilar, A., Martín-Gómez, S. \& San Nicolás-Santos, M. B. (2020). Análisis de las políticas de enseñanza universitaria en España en tiempos de Covid-19. La presencialidad adaptada. Revista De Educación a Distancia (RED), 21, 65. doi:10.6018/red.450461 
MELÉNDEZ \& SEDEÑO/Innovación educativa en tiempos de Covid-19 en el proyecto Galería Central: una experiencia de creatividad docente en comunicación del arte

Barbosa Becerra de Souza, B. (2009). Hibridación y transdisciplinariedad en las artes plásticas. Educatio Siglo XXI, 27, 1, 217-230. Disponible en: https://revistas.um.es/ educatio/article/view/71151

Buck-Morss, S. (2004). Estudios visuales e imaginación global. Antípoda, 9, 19-46.

Bourriaud, N. (2004). Posproducción. Buenos Aires: Adriana Hidalgo.

Chipia, J. (2013). Redes sociales virtuales para la educación y el cambio del ocio digital al ocio productivo. Revista Eduweb, 7, 1, 129-140.

Cortes, P. \& Márquez, M. J. (2017). Creatividad, comunicación y educación: Más

allá de las fronteras del saber establecido. Málaga: UMA Editorial.

Estrada Molina, O., Fuentes Cancell, D. R. \& García Hernández, A. (2021). Engajamento na educação virtual: experiências durante a pandemia de COVID-19. Texto Livre: Linguagem e Tecnologia, Belo Horizonte-MG, 14(2), 1-18. doi:10.35699/1983-3652.2021.33936

Faramarzi, S., Heidari, H. \& Chalak, A. (2019). Telegram: an instant messaging application to assist distance language learning (App Review). Teaching English with Technology, 19(1), 132-147.

Feldman, J. (2012). Las múltiples caras del curador. III Jornadas de estudiantes y graduados en Crítica de Artes. Buenos Aires: IUNA.

Franco Campoverde, Y.L. \& Malagón Rosado, P.E. (2021). Uso de Telegram como gestor educativo y su incidencia en el aprendizaje virtual. Creación de Manual Digital para uso de Telegram como herramienta educativa. Trabajo fin de Grado de Universidad de Guayaquil, Facultad de Filosofía, Letras y Ciencias de la Educación. http://repositorio.ug.edu.ec/ handle/redug/53382

Gazca Herrera, L. A. (2020). Implicaciones del coronavirus covid-19 en los procesos de enseñanza en la educación superior. RIDE Revista Iberoamericana para la investigación y el desarrollo educativo, 11, 21. doi:10.23913/ride.v11i21.753

Hodges, Ch., Moore, S., Lockee, B., Trust, T. \& Bond, A. (2020, March 27). The Difference Between Emergency Remote Teaching and Online Learning. Educause Review. Disponible en: https://bit.ly/3h6Bjh5

Iksan, Z. \& Mohd, S. (2017). Mobile learning: innovation in teaching and learning using Telegram. International Journal of Pedagogy and Teacher Education, 1, 1, 19-26. doi:10.20961/ijpte.v1i1.5120

Izquierdo Expósito, V. \& Fernández Maestre, M. (2019). Documentación para el periodista especializado en arte: la importancia de las fuentes. Documentación de las Ciencias de la Información, 42, 105-115. doi:10.5209/dcin.63087

Jiménez, Y. \& Ruiz, M. A. (2021). Reflections on the Challenges that Higher Education 
Faces in Times of COVID-19. Economía y Desarrollo, 165, 1. http://scielo.sld.cu/scielo. php?script=sci_arttext\&pid=S0252-85842021000200003\&lng=es\&tlng=en

Kasa, M. G. \& Yusuf, A. (2020). Experience of an Academic Library during the covid-19 pandemic. Library Philosophy and Practice (e-journal). 4456. https://digitalcommons.unl. edu/libphilprac/4456

Koldobsky, D. (2009). Nuevas figuras de a utor en las a rtes y en los medios. Proyecto ACyT. Buenos Aires: Instituto Universitario Nacional del Arte.

Lin, C.-Y., \& Reigeluth, C. M. (2021). Orientación para el aprendizaje colaborativo apoyado en wiki y construcción de conocimiento comunitario para una clase completa: Cómo mejorar los entornos de aprendizaje durante la pandemia COVID19. Revista De Educación a Distancia (RED), 21, 65. doi:10.6018/red.447401

Lumbreras, T. Garnelo, I. \& Gabilondo, V. (2011). Galería Central: un proyecto de innovación colaborativo. Comunicación presentada a CINDU, IV Congreso Internacional de Docencia Universitaria CINDU 2015, El futuro de la docencia en la universidad. Vigo.

Mansell, R. (ed.) (2003). La revolución de la comunicación. Madrid: Alianza.

Martínez-Nicolás, M. Saperas E. \& Humanes, M.L. (2019). Mudanzas en la cultura científica. El nuevo contexto de la practicas investigadora sobre comunicación y sus implicaciones epistemológicas. En Epistemología de la comunicación y cultura digital: retos emergentes (pp. 23-41). Granada: Eug.

Ministerio de Universidades (2020). Recomendaciones del Ministerio de Universidades a la comunidad universitaria para adaptar el curso universitario 2020-2021 a una presencialidad adaptada y medidas de actuación de las universidades ante un caso sospechoso o uno positivo de Covid-19. Ministerio de Universidades del Gobierno de España. Disponible en: https://www.ciencia.gob.es/stfls/MICINN/Universidades/Ficheros/Recomendaciones_ del_Ministerio_de_Universidades_para_adaptar_curso.pdf

O’Neill, P. \& Wilson, M. (2010). Curating and the Educational Turn. London \& Amsterdam: Open Editions and De Appel.

Ovejero, A. (1988). Psicología Social de la Educación. Barcelona: Herder.

Prendes Espinosa, M. P. \& González Calatayud, V. (2020). Acoso y ciberacoso en la escuela: la vulnerabilidad de las personas con necesidades educativas especiales. Barcelona: Octaedro.

Rodrigo-Montero, J. (2015). Kunstcoop: Experiencias de mediación artística en Alemania. Arte, Individuo y Sociedad, 27(3), 375-393. doi:10.5209/rev_ARIS.2015.v27.n3.43723

Rué, J., Font, A. \& Cebrián, G. (2011). El ABP, un enfoque estratégico para la formación en Educación. Aportaciones de un análisis de la formación en Derecho. REDU, Revista de Docencia Universitaria, 9(1), Enero - Abril, 25-44. Disponible en: http://grupcomplex. uab.cat/_docs/Cebrian_2011_El_ABP_un_enfoque_REDU.pdf 
MELÉNDEZ \& SEDEÑO/Innovación educativa en tiempos de Covid-19 en el proyecto Galería Central: una experiencia de creatividad docente en comunicación del arte

Salas, M. \& Salas, J. (2018). M-Learning - una experiencia colaborativa usando el software Telegram. Revista científica retos de la ciencia, 2(1), 85-94. Disponible en: https:// retosdelaciencia.com/Revistas/index.php/retos/article/view/205

Sánchez, F. (2018). Competencia digital: una necesidad del profesorado universitario en el siglo XXI. Revista de Educación a Distancia (RED), 56. doi:10.6018/red/56/7

Sanz-Daza Pérez, E. (2018). Manual del Comisario. En Galería Central 2012-2014 (pp. 12-36). Málaga: Universidad de Málaga.

Sedeño-Valdellos, A. (2019). Investigación artística en comunicación: territorios comunes e innovación metodológica en la hibridación entre arte y comunicación En Memorias de Congreso $2 o$ Congresso Internacional Media Ecology and Image Studies. O protagonismo da narrativa imagética (pp. 946-969). Portugal: Ria Editorial.

Tirado, A. (2019). La exposición de arte desde la transferencia del conocimiento artístico. Educación Artística: Revista de Investigación, 10, 168-183. doi: 10.7203/eari.10.13979

UNESCO (2006). Hoja de ruta para la educación artística. Conferencia Mundial sobre la Educación Artística: construir capacidades creativas para el siglo XXI. Disponible en: http://www.unesco.org/new/fileadmin/MULTIMEDIA/HQ/CLT/CLT/pdf/Arts_Edu_ RoadMap_es.pdf. 